\title{
August Ludwig Schlözer zwischen Petersburg und Göttingen
}

\author{
Reinhard LAUER
}

Die Akademie der Wissenschaften zu Göttingen begeht mit der heutigen Feier den 200. Todestag von August Ludwig von Schlözer. Sie tut das in dem Wissen, daß Schlözer niemals Ordentliches Mitglied dieser Institution, besser: der Königlichen Societät der Wissenschaft, wie sie zu Schlözers Lebzeiten hieß, gewesen ist. Zwar war er bereits mit 26 Jahren zum Korrespondierenden Mitglied der Societät und 1766 zum Auswärtigen Mitglied gewählt worden, die Wahl zum Ordentlichen Mitglied jedoch geriet zu einem Skandal, der die gesamte Akademie, 18 Jahre nach der Gründung, in ihrem Bestand gefährdete. Schlözer, einer der berühmtesten Professoren der Göttinger Universität, in Göttingen als Universitätslehrer bei den Studenten beliebt und geschätzt; Schlözer, zu seiner Zeit in ganz Deutschland durch seine Zeitschriften bekannt; Schlözer, nach einhelligem Urteil der bedeutendste Historiker des 18. Jahrhunderts, der Begründer einer ganzen Reihe neuer wissenschaftlicher Disziplinen, - August Ludwig Schlözer also ist ein Beispiel dafür, daß in gewissen Fällen auch die Besten nicht in die Societät bzw. Akademie gewählt wurden und werden. Wie ist das möglich? Es ist möglich aus verschiedenen Gründen, von denen ich hier wenigstens vier nennen möchte:

1. Es wurden und werden bestimmte wissenschaftliche Disziplinen nicht in der Akademie vertreten, lange Zeit die Medizin, die Wirtschafts- und Sozialwissenschaften, die Forst- und Agrarwissenschaften und andere.

2. Es kann sein, daß das Fach, das ein Gelehrter vertritt, neu ist oder nicht an der Akademie ausgewiesen ist, so daß sich niemand findet, der den Vorschlag zur Wahl unterbreiten kann. Das geschieht immer wieder, auch gerade zur Zeit könnten einschlägige Fälle genannt werden.

3. Es kann vorkommen, daß ein bedeutender Gelehrter so still und bescheiden vor sich hinforscht, daß er den Kollegen nicht auffällt und nicht in die Akademie gewählt wird. Das mag selten sein, ist aber denkbar. Bei Schlözer war es nicht der Fall.

4. Der bei weitem häufigste Grund aber ist einfach Neid, menschliche Mißgunst, Streit zwischen den Kollegen, der sich auf den Vorschlagenden oder auf den Vorgeschlagenen beziehen kann. In einer hiervon bestimm- 
ten Situation wurde die Wahl Schlözers zum Ordentlichen Mitglied 1769 vereitelt. Davon wird noch die Rede sein.

Schlözer stammte aus einem protestantischen Pfarrhaus; er wurde am 5. Juli 1735 in Gaggstadt bei Kirchberg, im Hohenlohischen, geboren und ging 1751 zum Theologiestudium nach Wittenberg. Da aber einer seiner Lehrer, der Theologe Hoffmann, ständig gegen David Michaelis in Göttingen polemisierte, weil der das Alte Testament mittels der philologisch-kritischen Methode behandelte, wechselte Schlözer 1754 nach Göttingen und geriet nun ganz in den Bann des in Wittenberg geschmähten Michaelis. Dieser erkannte sofort die vielseitige Begabung und ungewöhnliche Tatkraft Schlözers und sah in ihm den Mann, der durch eine Orientexpedition neue Aufschlüsse über die Welt des Alten Testaments erbringen sollte. Es ist jene Orientexpedition, die nach Michaelis' Vorstellungen im Auftrag des dänischen Königs in den Jahren 1761-67 von Carsten Niebuhr und weiteren Wissenschaftlern, die Michaelis ausgewählt hatte, durchgeführt wurde. Schlözer war nicht unter ihnen.

Dabei hatte sich Schlözer jahrelang systematisch auf diese Expedition, sein immer wieder beschworenes Orientprojekt, vorbereitet, indem er alte und neue Sprachen lernte, sich mit Politik, Geschichte, Statistik, Ökonomie, Münzwesen, Naturwissenschaften und der Linnéschen Botanik beschäftigte. 1756 ging er als Hauslehrer nach Schweden, war dann wieder in Göttingen und erhielt 1761 durch die Vermittlung des Geographen Büsching eine Stelle als Gehilfe des russischen Reichshistoriographen Gerhard Friedrich Müller in Sankt-Petersburg.

Was zunächst nur als das willkommene Sprungbrett zur Verwirklichung der Orientreise gedacht war - Schlözer beabsichtigte, über Südrußland in das Osmanische Reich einzureisen, nicht auf dem üblichen Wege über Istanbul -, trieb ihn bald in ganz andere Bahnen. Schlözer erlernte, aufgrund seiner 15 fach erprobten sogenannten „Wurzelmethode“ des Spracherwerbs, überaus rasch die russische und die kirchenslavische Sprache und beschäftigte sich mit den von Müller zusammengetragenen altrussischen Rechtsdenkmälern und Chroniken. Da die Chroniken bis dahin so gut wie unbearbeitet geblieben waren, bot sich eine kritische Sichtung nachgerade an. Schlözer sah, daß sich hier ein neues, unbeackertes Arbeitsfeld eröffnete, das ihn die früheren Pläne vergessen ließ. Seine Stellung als schlechtbezahlter Adjunkt der Kaiserlichen Akademie der Wissenschaften zu Sankt-Petersburg war alles andere als glänzend. Hinzu kam, daß er sich mit dem mächtigsten Mann der Akademie, dem Universalgelehrten und Dichter Michail Lomonosov, zerstritt. 1764 ging seine rasch niederge- 
schriebene russische Grammatik in Druck, deren Erscheinen jedoch durch Lomonosov unterbunden wurde. Lomonosov, beunruhigt durch den ehrgeizigen jungen Deutschen, hatte einfaches Spiel mit ihm; denn Schlözer hatte fälschlicherweise das russische Wort bojarin, der Bojare' von baran ,der Hammel' und das Wort für ,Fürst' - knjaz' - von dem deutschen Knecht abgeleitet. Damit hatte er mächtige Würdenträger des Reiches gegen sich aufgebracht. Schlözer sann nun darauf, sein Renommé im Ausland zu heben. Und dabei war ihm Michaelis nach Kräften behilflich.

Schon 1761 war Schlözer, ganze 26 Jahre alt, zum Korrespondierenden Mitglied der Göttinger Societät gewählt worden. Im Juli 1763 bat er seinen Lehrer Michaelis ziemlich unverblümt, ihm bei der Hannoverschen Regierung ein Prädicat, d.h. eine Scheinberufung (sie entspräche heute etwa einer apl. Professur) zu verschaffen, von der er sich in Petersburg günstigere Bedingungen erhoffte. Michaelis ließ sich auf dieses Ansinnen ein, indem er dem Hannoverschen Kabinett versicherte, daß man von Schlözer „viel Ehre und eine überaus vorteilhafte Correspondenz" erwarten könne. Er verschwieg aber auch nicht die problematischen Charaktereigenschaften seines Schützlings; und das liest sich in einem Brief vom 2. September 1763 an den Kurator Baron von Münchhausen so:

Sein Gemüth anlangend, so hat er einen überaus großen Eigensinn, der alle Gefahr affrontirt, aber auch oft zum großen Fehler wird. Er hat sich bisher unter denen, die mit ihm in näherer Connexion gestanden, beynahe blos mit mir vertragen können. Sein Gesicht hat eine kenntliche Ähnlichkeit mit Carl dem 12ten, und ohngefähr so sieht auch seine Seele aus. Selbst die niedergeschlagenen Augen und das blöde im Reden von diesem Helden hat die Natur an ihm imitirt. Sein Eigensinn und große Courage macht ihn zu einer Reise [Anspielung auf das Orientprojekt] so bequem, als unter 10.000 Gelehrten nicht einer seyn wird. Allein eben der Eigensinn macht, daß ich ihn nicht eigentlich recommandire, sondern blos unterthänigst anfrage, was E. Exc. zu seinem Gesuch denken: indem ich nicht gern Verantwortung haben möchte, wenn er auch hier eigensinnig wäre, und darüber Schade entstünde.

So ungewöhnlich der Vorschlag war, er wurde aufgenommen, und im April 1764 verlieh der englische König Schlözer das Prädicat eines Professors ad honores in Göttingen, ein Pfund, mit dem er nun wuchern konnte. Umtriebig machte sich Schlözer an die Auswertung der altrussischen Chroniken. Um seine Stellung in Sankt-Petersburg zu verbessern, legte er im Juni 1764 mit 28 Jahren - der Petersburger Akademie einen Arbeitsplan zur Erstellung einer Russischen Reichsgeschichte unter dem Titel „Gedanken über die Art, die russische Historie zu traktieren“" vor. Hier schlug er vor

1. eine systematische Erforschung der einheimischen Denkmäler,

2. die Erforschung der ausländischen Denkmäler und 
3. die Auswertung beider zur Erstellung einer russischen Reichsgeschichte, einer „schönen Geschichte“, wie er das nannte.

Um diese Pläne, besonders den zweiten, zu verwirklichen, wandte er sich mit einer Bittschrift unmittelbar an die Kaiserin Katharina II., die vom Stil und der präzisen Art des Antrages so angetan war, daß sie Schlözer nicht nur eine Reise nach Göttingen gewährte und den Besitz der von Schlözer gesammelten Quellen bestätigte, sondern ihn darüber hinaus auch noch zum Ordentlichen Professor der russischen Geschichte auf sieben Jahre, zu günstigsten, neiderregenden Bedingungen ernannte. Zugleich wurde Schlözer Ordentliches Mitglied der Petersburger Akademie. Katharina stellte sich damit im Streit zwischen Lomonosov und Schlözer eindeutig hinter Schlözer. Schon im Januar 1765 erhielt er seine Bestallung.

Schlözer hat dies seiner Förderin vielfältig vergolten, vor allem durch seine Schrift „Neuverändertes Rußland“ (1767), für die er bereits im Juni 1765 von der Akademie „authentische Dokumente“ über die Regierungstätigkeit der Kaiserin anforderte. Die Dinge liefen für ihn gut in Rußland. In einem Brief an den Akademiesekretär Taubert fomulierte er es so:

Die alte russische Historie, sagt meine Kaiserin in meinem Kontrakt, soll meine Hauptbeschäftigung sein. Diese Historie hat 2 Teile, einen so groß wie den anderen, einen ausländischen und einen inländischen.

Seine Urlaubsreise nach Deutschland trat er im Juni 1765 an, er nutzte sie weidlich, seine Rückkehr nach Göttingen zu betreiben.

Im Februar 1766 wurde er von der Göttinger Philosophischen Fakultät honoris causa promoviert; kurz vor Antritt der Rückreise nach Rußland wurde er zum Auswärtigen Mitglied der Göttinger Societät gewählt, wo er am 14. Juni 1766 seinen Vorstellungsvortrag über die slavische Geschichte in lateinischer Sprache unter dem Titel „Memoriae Slavicae“ hielt. Das heißt: Schlözer stellte sich der Societät ausdrücklich mit einem slavischen Thema vor. Es war dies der erste slavistische Vortrag in der Göttinger Akademie. Schlözer wollte den Sodalen mit ihm seine neue, kritische Methode vorzuführen. Erlauben Sie mir, dies in Kürze anzusprechen.

Es geht in diesem Akademievortrag um die Widerlegung des Irrtums derjenigen, „welche die Ursitze der Slaven in den Gegenden des Kaukasus, zwischen dem Schwarzen und dem Kaspischen Meer, annehmen“. Dabei steht im Vordergrund nicht so sehr die Frage, welches der alten Völker mit den Slaven identisch ist - Schlözer deutet nur an, daß es die bei Ptolemäus, Plinius und Tacitus erwähnten Veneder gewesen sein mögen -, als vielmehr die Demonstration kritischer Methode. In der Herkunftsfrage 
erkennt Schlözer drei Hauptklassen von Mutmaßungen, die zu widerlegen er sich anschickt:

1. die Meinung, Skythen und Sarmaten seien mit den Slaven identisch, also „uno ovo";

2. die Stammvater- oder Anführertheorie (Eponymtheorie), die besagt, daß die einzelnen slavischen Völker jeweils von einem Stammvater ihren Namen erhalten haben;

3. die etymologische Methode, die unterschiedliche, getrennte Völker aufgrund des zufälligen Gleichklangs der Namen in einen genetischen $\mathrm{Zu}-$ sammenhang bringt.

In der Vorlesung nun werden in kritischer Argumentation nicht nur die Thesen selbst, sondern auch die Methoden, die sie stützen, zurückgewiesen. Hinsichtlich der Skythen- und Sarmatentheorie kann Schlözer unter Berufung auf den Petersburger Historiker Gottlieb Siegfried Bayer zeigen, daß die Alten im allgemeinen nur äußerst ungenaue Vorstellungen von den „nördlichen“ Völkern besaßen. Die für verschiedene slavische Völker bzw. Städte angenommenen "Stammväter" - die Russen stammen von Rus ab, die Lechen oder Polen von Lech, Krakau von Krak, die Tschechen von Czech, die Kroaten von Horvat usf. - sind reine Erfindungen, wie sie auch schon in den Geschichtsdarstellungen der Griechen begegneten. Und über das Etymologisieren, oder besser: die Etymologisiersucht, fällt Schlözer endlich ein vernichtendes Urteil. Er wendet sich vor allem gegen die These, die Polen hätten ihr Stammvolk in den aus der Kolchis bekannten Laziern (Lazai), ebenso wie gegen die These, die Zichen, ein Steppenvolks im nördlichen Kaukasus, seien die Vorläufer der Tschechen. Der zufällige Anklang der Völkernamen (Lazii - Polacy, Zichen - Tschechen) hatte einige Gelehrte (Dobner, Lengnich) zu der abstrusen Annahme einer kaukasischen bzw. kolchischen Herkunft der Slaven geführt. Daß dabei die Zichen mit den Cherkessen verwechselt wurden, zwei Völker, die nie slavisch gesprochen haben, kann Schlözer leicht abtun. Am Beispiel der angeblichen Beziehung Lacii - Polacy zeigt er dann den ganzen Unfug des Etymologisierens auf, denn Polacy, der Nominativ Pluralis von Polak, der Pole ist natürlich von dem Stamm pol- ,Feld, Ebene' und dem Suffix -ak gebildet, mit anderen Worten: es gibt überhaupt gar kein Etymon -lac-, das eine Verbindung zwischen Polen und Laziern zuließe. So genügte allein die Kenntnis einfachster Regeln der polnischen Wortbildung, um den Irrtum auszuräumen.

Die Warnung vor dem Etymologisieren gehört denn auch zu den Grundpostulaten, die Schlözer Historikern und Philologen nachdrücklich vor Augen hält. Vielleicht erinnerte er sich an die Schwierigkeiten, die seiner- 
zeit in Petersburg seine eigenen Pseudoetymologien barin von baran und knjaz' von Knecht hervorgerufen hatten. Noch in den methodologischen Resümees der Nestor-Ausgabe heißt es:

Gewönen sich auch die Herren das Etymologisiren ab, sowie sie es noch immer fast one Ausnahme treiben. Es ist in unseren hellen historischen Tagen allzu verächtlich.

Doch mit dem Vortrag und der Wahl zum Auswärtigen Mitglied der Gelehrten Gesellschaft war die Angelegenheit noch nicht beendet. Schon im folgenden Jahr, im Herbst 1767, wurde Schlözer erneut eine Reise nach Deutschland gewährt. Diesmal brachte er vorsichtshalber in seinem Reisegepäck zwei Folianten mit, die seine Exzerpte aus zehn altrussischen Chroniken enthielten. Ohne Zweifel hatte er insgeheim die Absicht, nach Auslaufen seines Kontraktes Rußland zu verlassen und nicht mehr zurückzukehren. Um seinen wissenschaftlichen Rang zu demonstrieren - bisher hatte er vorwiegend auf Kredit gelebt -, legte er in kurzen Abständen eine Reihe bemerkenswerter rußlandkundlicher Werke vor: „Tableau de l'histoire de la Russie“ (1767), ein Überblick über die russische Geschichte; „Probe russischer Annalen“ (1768), ein Aufriß über die Methode der Darstellung der russischen Chronik; „Annales Russici“, eine erste Probe des Chronikvergleichs; das „Neuveränderte Rußland oder Leben Catharinae der Zweyten Kaiserinn von Rußland“ (1767, drei Auflagen bis 1771), eine statistische, noch heute gültige Beschreibung des Beamtenstaates des Katharinensischen Rußland, sowie eine Schrift „Von der Unschädlichkeit der Pocken in Rußland und von Rußlands Bevölkerung überhaupt" (1768), wo aus Anlaß der Pockenimpfung, die ein besonderes Anliegen Katharinas war, demographische Methoden entwickelt werden, die am Anfang der russischen Bevölkerungsstatistik stehen. Man könnte hinzuzählen die „Darstellung der Allgemeinen Nordischen Geschichte", als 31. Teil der Welthistorie, sein Hauptwerk in dieser Zeit, an dem er mehrere Jahre gearbeitet hatte. Dieses Werk brachte eine klassifikatorische Aufführung der „slavischen Geschichten“ und „slavischen Sprachen“, genauer: ein Linnésches System der slavischen Völker und Sprachen. Er war jetzt in der russischen Geschichte und in der russischen Statistik auf unerreichter Höhe, wobei nach seiner Auffassung die Statistik als eine stillstehende Geschichte und die Geschichte als eine fortlaufende Statistik anzusehen war.

Mit seinen Schriften, so hoffte er, könne er womöglich den Status eines Auswärtigen Mitgliedes der Petersburger Akademie mit Rang und Gehalt eines Kollegien- oder Hofrates (d.h. der 6. oder der 7. Rangklasse der Petrinischen Rangtabelle) erlangen. Damit wäre, wie es Wilhelm Schwarz in seiner Schlözer-Biographie ausdrückt, in Göttingen eine Art „Außenstelle“ 
der Petersburger Akademie eingerichtet worden. Graf Orlov, der Präsident der Petersburger Akademie, hielt es nicht für tunlich, auf dieses Ansinnen überhaupt zu antworten. Statt seiner erteilte Jakob Stählin, der Konferenzsekretär der Akademie, den abschlägigen Bescheid: Das Anerbieten müsse abgelehnt werden, hieß es in dem Brief, weil sich (sonst) jedes Mitglied das Glück gewünscht hätte, seine Besoldung in Deutschland zu verzehren. In der gleichen Angelegenheit schrieb Stählin maliziös an Gerhard Friedrich Müller (9. Februar 1769):

Ich bin recht begierig auf seine (Schlözers) Antwort [...] über öfteres Brustfieber u. fast beständige Kräncklichkeit klagt er auf allen seiten seiner Briefe. Was wird mein Brief, der von Wort zu Wort aus dem Academischen Protocoll geschrieben ist, bey solchem Zustand ausrichten? Wenn er ihm nur nicht gar den Schlagfluß macht!

Schlözer aber gab sich noch im Juli 1769 der Hoffnung hin, man werde ihn in Petersburg halten. Er schrieb an Johann Albrecht Euler, den neuen Ständigen Sekretär der Akademie, er sei

noch in statu (also: in Stellung), und alsdann ist es noch Zeit zu bestimmen was Katharina II. mit ihrem künftig freiwilligen Biographen und die Kaiserliche Akademie mit ihrem künftig freiwilligen Historico machen will.

Anders reagierte man in Hannover. Hier verfolgte der aufgeschlossene Kurator Münchhausen mit lebhaftem Interesse die rußlandkundlichen Studien Schlözers und war, als Schlözer in Gnaden aus russischen Diensten entlassen wurde, gern bereit, ihn als o. Professor der Philosophischen Fakultät zu berufen. Der englische König drückte sich in einem Schreiben an seine Räte in Hannover höchst positiv über Schlözer aus und stimmte der Berufung zu:

Da dieser mann von einer solchen Seite bekannt ist, daß ihr vermeynet, daß er der Universitaet vielen Vortheil bringen, und bey derselben mit Nutzen zu gebrauchen seyn werde, er auch den Ruf anzunehmen geneigt ist, so genehmigen Wir, daß er mit einer Besoldung von fünf huntert Rthlr aus der Universitaets-Casse, zum Professor ordinarius in der philosophischen Facultaet bestallet werde.

Das Schreiben war am 30. Mai 1769 reskribiert und am am 6. Juni 1769 empfangen worden. Die Ernennung erfolgte am 14. Juni 1769, auf den Tag genau drei Jahre nach der Antrittsvorlesung in der Societät. Im Vorspann zur Nestor-Edition hat Schlözer später berichtet, daß Münchhausen mit der Berufung die Erwartung verbunden habe, er werde künftig die russische Literatur in Deutschland bekannt machen. So wurden ihm drei „slavistische“ Aufgaben gestellt: 
1. die Ausgabe der russischen Annalen (altrussischen Chroniken) zu besorgen - hierfür sollte gegebenenfalls eine russische Druckerei angeschafft werden;

2. einzelne Abhandlungen für die Göttinger Societät der Wissenschaften zu verfassen, und

3. für die „Göttingischen Gelehrten Anzeigen“ russische Bücher zu rezensieren.

Daß Schlözer diese Aufgabe dann bis etwa 1800 zurückstellte und sich fast ganz der Statistik, Politik, Universalgeschichte und Pädagogik widmete, lag daran, daß Michaelis, der Direktor der Königlichen Societät der Wissenschaften, mit dem Versuch scheiterte, Schlözer mit seiner Berufung sogleich auch zum Ordentlichen Mitglied der Societät (der heutigen Akademie der Wissenschaften) zu machen. Michaelis war mit fast allen Sodalen zerstritten, vor allem mit dem Mathematiker Abraham Gotthelf Kästner und dem Philologen Christian Gottlob Heyne. Kästner, der boshafte Mathematiker und Dichter, hatte Schlözer rasch aufs Korn genommen. Er hatte böse Epigramme gegen Schlözer verfaßt, die ins Persönliche gingen. Schlözer beschwerte sich bei der Landesregierung über den „Pasquillenunfug“. Es gab auch Gelehrtenstreit zwischen beiden, etwa um die Frage der Herkunft des „Lech“ oder über die Herstellung der Historischen Klasse (gegen die Mathematische) an der Petersburger Akademie. Kästner lästerte über Schlözers Heirat mit der Tochter des verstorbenen Leibmedikus Röderer usw. Am stärksten aber wirkte die Unterstellung, Schlözer habe seine Göttinger Professur nicht aufgrund seiner Leistungen, sondern nur durch Beziehungen und Kontakte erhalten. Kästner und Heyne sprachen sich dezidiert gegen die Wahl Schlözers als Ordentliches Mitglied der Societät aus. Für Schlözer optierten am Ende lediglich Michaelis und der Theologe Walch. Das Scheitern der Wahl Schlözers löste die erste große Krise der Göttinger Akademie nach ihrer Gründung aus. Michaelis trat verbittert von seinem Amt als Ständiger Direktor der Königlichen Gesellschaft zurück, womit seine Mitgliedschaft erlosch; das Gleiche tat der Historiker Johann Philipp Murray, der das Sekretärsamt wahrgenommen hatte. Michaelis war ein selbstbewußter, eigenwilliger Direktor gewesen. Es heißt, daß allgemein Erleichterung geherrscht habe, als nach Michaelis der Philologe Heyne das Heft in die Hand nahm. Der Jurist Ferdinand Frensdorff hat 1892 in einer ausführlichen Studie diese „Krisis in der Königlichen Gesellschaft der Wissenschaften" dargestellt und gezeigt, daß es eine Bestandskrise war. Ihre Überwindung brachte die neuerliche Konsolidierung - allerdings ohne Schlözer. 
Schlözer war mit diesem unerfreulichen Akt die reguläre Mitarbeit in der Societät und ihren Publikationen, den „Anzeigen von gelehrten Sachen“ bzw. den „Göttingischen Gelehrten Anzeigen“, verwehrt; auch seine Verbindungen nach Petersburg waren zunächst gestört. Münchhausen starb 1770 und konnte nichts mehr ins Lot bringen. Nach dem Tod Achenwalls, des berühmten Statistikers, wurde Schlözer angetragen, die Statistik zu lesen. Schlözer feierte in diesem neuen Fach bald triumphale Erfolge. So wurde aus dem Slavenkundler, der eigentlich als Theologe begonnen hatte, nunmehr der Statistiker und Politologe. Zu einer europäischen Berühmtheit wurde Schlözer ferner durch seine politischen Zeitschriften: „Briefwechsel meist statistischen Inhalts“ (1774/75); „Briefwechsel meist historischen und politischen Inhalts" (1776-82) und "Staatsanzeiger" (1783-95).

Doch dann geschah ein Wunder: Seit etwa 1800 widmete sich Schlözer erneut und nun fast ausschließlich den slavischen Dingen. Vor allem die große Edition der altrussischen Nestor-Chronik wurde nun vorangebracht. In den Jahren 1802-1809 legte er sie in fünf Bänden vor. Der erste Band, dem neuen Zaren Alexander I. gewidmet, brachte Schlözer den VladimirOrden und damit den erblichen russischen Adel ein. Erst von jetzt an hieß er August Ludwig von Schlözer. Wenige Monate nach Erscheinen des letzten Bandes der Nestor-Chronik ist Schlözer gestorben.

Schlözers Rückkehr zur Slavenkunde hat verschiedene Gründe: 1795 wurde ihm nach einer politischen Affäre - er hatte die Leibeigenschaft in Kurpfalz kritisiert - die politisch-publizistische Tätigkeit seitens der Hannoverschen Regierung untersagt, so daß er sich um so stärker wieder seinen Vorlesungen zuwandte, namentlich der Nordischen Geschichte und der Statistik. Nach dem Regierungsantritt Alexanders I. sammelten sich in Göttingen russische Studenten, die vornehmlich um seinetwillen hierhergekommen waren, um ihn über die russische Geschichte dozieren zu hören. Der tiefere Grund aber ist wohl der, daß für ihn - wie er einmal sagte - die russische Geschichte sein „Feuer und Herd“, sein „Monopol“ war. Der Nestor sei, versicherte er, all die Jahre zwischendurch nie von seinem Pulte gekommen; er habe sich „ein Menschenalter hindurch, ohne speciellen Beruf, und nur in den Nebenstunden, aber con amore" den Nestor-Studien gewidmet.

So sehen wir in Schlözers Gelehrtenleben zwei Phasen, in denen er sich mit größter Energie den slavischen Angelegenheiten widmete: zuerst 1762 1770, als er zwischen St. Petersburg und Göttingen hin- und herpendelte; dann von 1800 bis zu seinem Tod 1809 in Göttingen, jedoch in engem Kontakt mit russischen Studenten. Er war inzwischen zu alt, um noch, etwa als Reichshistoriograph, nach Petersburg berufen zu werden. 
Seinen Göttinger Vortrag von 1766, der eine erste Probe seiner kritischen Wissenschaft bot, hatte Schlözer in einem Augenblick gehalten, da es noch so aussehen konnte, als werde sein Ansinnen, sowohl in Sankt-Petersburg als auch in Göttingen zu wirken - also auch mit einem doppelten Gehalt versehen zu sein -, verwirklicht werden. Es ist anders gekommen. Schlözer blieb am Ende nur Göttingen als Wirkungsort. Die Absage der Königlichen Societät der Wissenschaften, ihn als Ordentliches Mitglied in ihre Reihen aufzunehmen, ließen die slavistischen Aufgaben, die Münchhausen ihm zugedacht hatte, zunächst in den Hintergrund treten. Seine Erfolge in der Statistikvorlesung und als Zeitschriftenherausgeber trieben ihn auf andere einträgliche Felder. Schlözer zwischen Sankt-Petersburg und Göttingen das war nur eine Episode in seiner Biographie. Jedoch bildete die NestorEdition, die Wiederaufnahme der editorischen Versuche aus jenen Jahren, den krönenden Abschluß seines Lebens. 